\title{
Interacción entre crecimiento económico, estabilidad e inclusión financiera: evidencia empírica internacional
}

\author{
Interaction between economic growth, stability and financial inclusion: \\ International empirical evidence
}

\author{
Tomás Gómez Rodríguez*1, Humberto Ríos Bolívar², \\ Adriana Zambrano Reyes ${ }^{1}$ \\ ${ }^{1}$ Universidad Autónoma del Estado de Hidalgo, México \\ ${ }^{2}$ Instituto Politécnico Nacional, México
}

Recibido el 14 de marzo de 2019; aceptado el 3 de diciembre de 2019

Disponible en Internet el: 11 de diciembre de 2019

\section{Resumen}

Empleando una muestra de 71 países y un período que abarca desde 2007 al 2016. Se analiza la relación entre inclusión financiera y crecimiento económico, así como la relación entre estabilidad financiera y crecimiento económico empleando el método de Mínimos Cuadrados Ordinarios (MCO) con efectos fijos de dos vías. Así mismo, como prueba de robustez se emplea el Método Generalizado de Momentos (GMM) con efectos fijos de dos vías. Además, se realiza la prueba de causalidad de Granger con el método de datos apilados desarrollado por Dumitrescu y Hurlin (2012). Los resultados sugieren la existencia de un nexo negativo tanto para la relación entre inclusión financiera y crecimiento económico, así como para la relación entre estabilidad financiera y crecimiento económico. Los resultados de la prueba de causalidad exhiben que la relación se extiende desde las variables de inclusión financiera hacia el crecimiento económico. Mientras que la relación para las variables de estabilidad se extiende desde el crecimiento económico hacia estas variables.

Código JEL: D23, E44, O40

Palabras clave: Panel de datos; Mercados financieros; Crecimiento económico

* Autor para correspondencia

Correo electrónico ya1mx@yahoo.com.mx (T. Gómez Rodríguez).

La revisión por pares es responsabilidad de la Universidad Nacional Autónoma de México. 


\begin{abstract}
Using a sample of 71 countries and a period that covers from 2007 to 2016 . The relationship between financial inclusion and economic growth is analyzed, as well as the relationship between financial stability and economic growth using the Ordinary Least Squares (OLS) method with two-way fixed effects. Likewise, the Generalized Method of Moments (GMM) with two-way fixed effects is used as a robustness test. In addition, the Granger causality test is performed with the stacked data method developed by Dumitrescu y Hurlin (2012). The results suggest the existence of a negative link both for the relationship between financial inclusion and economic growth, as well as for the relationship between financial stability and economic growth. The results of the causality test show that the relationship extends from the variables of financial inclusion to economic growth. While the relationship for stability variables extends from economic growth to these variables.
\end{abstract}

JEL code: $\mathrm{C} 23, \mathrm{E} 44, \mathrm{O} 40$

Keywords: Panel data; Financial markets; Economic growth

\title{
Introducción
}

En la actualidad la literatura acerca de la relación entre desarrollo del sistema financiero y el crecimiento económico es extensa. Sin embargo, la investigación sobre cuál podría ser el efecto de las diferentes dimensiones del desarrollo financiero sobre el crecimiento económico se encuentra en sus primeras etapas. Entre estas dimensiones del sistema financiero se encuentran la estabilidad e inclusión financiera. La inclusión financiera implica que los adultos tienen acceso y pueden hacer uso efectivo de una gama de servicios financieros apropiados como: créditos, seguros y oportunidades de ahorro. Estos servicios deben ser proporcionados de una manera justa, equitativa y transparente a un costo accesible para todos los segmentos de la población. En su nivel más básico, la inclusión financiera significa tener una cuenta para realizar transacciones o una cuenta de depósito en un banco u otra institución financiera o a través de un proveedor de servicios de dinero móvil (Sethi y Acharya, 2018). En el año 2014, 2 billones o 38 por ciento de los adultos no tenían acceso a dicha cuenta, de acuerdo con Demirguc-Kunt, Klapper, Singer y van Oudheusden (2015).

Los posibles beneficios de la inclusión financiera son: promover el crecimiento económico, ayudar a disminuir la pobreza y reducir la desigualdad del ingreso, ayudar a las personas a invertir en el futuro, suavizar su consumo y administrar los riesgos financieros (Demirguc-Kunt, Klapper y Singer, 2017). Específicamente el acceso a los servicios fi- 
nancieros fomenta el crecimiento económico a través de impulsar la utilización de cuentas bancarias como alternativa a los pagos en efectivo, esto permite que las transacciones sean más eficientes, transparentes y seguras. También ayuda a las personas en estado de pobreza a superar esta situación al hacer posible la inversión en educación y negocios. Al facilitar formas de administrar los shocks en los ingresos como pueden ser el desempleo o la pérdida de un sostén de la familia, la inclusión financiera también puede prevenir que las personas caigan en la pobreza. Esto es especialmente relevante para las personas que vienen en los hogares más pobres (Demirguc-Kunt, Klapper y Singer, 2017). En sociedades con bajos niveles de inclusión, las personas deben afrontar mayores problemas económicos debido a la falta de servicios financieros. Particularmente la falta de acceso a los servicios financieros puede afectar negativamente al crecimiento económico debido a una deficiente infraestructura financiera de acuerdo con: Gurley y Shaw (1955), Goldsmith (1969), Diamond y Dybvig (1983), Greenwood y Jovanovic (1990) y Angadi, (2003). Más aún, la falta de inclusión puede llevar a la falta de educación financiera y al surgimiento de un sector financiero informal y no organizado (Sharma, 2016).

Mientras la inclusión financiera tiene beneficios como: generación de crecimiento, creación de trabajos, reducción de la pobreza, disminución de la desigualdad del ingreso y mejora de la calidad de vida. En contraste, un rápido y desregulado acceso a los servicios puede tener efectos adversos sobre el crecimiento económico. Esta situación se presenta cuando se alcanza la inclusión financiera a través de un aumento demasiado rápido del crecimiento del crédito a través de intermediarios no regulados, lo cual puede afectar la estabilidad financiera y como consecuencia puede resultar en un nexo negativo entre inclusión financiera y crecimiento económico. (Mehrotra y Yetman, 2015).

Por otro lado, una de las principales consecuencias de la existencia de bajos niveles de estabilidad puede ser la ocurrencia de crisis financieras. Estas crisis pueden tener un efecto dañino sobre el crecimiento económico y el bienestar social. Así mismo, estas crisis pueden afectar hasta a las economías más avanzadas, sin embargo, el daño que pueden causar en países con un ingreso bajo pueden ser aún más severo. Ya que las personas que viven en estos países, no tienen margen de maniobra para cubrirse del riesgo de malos resultados económicos y su modo de vida se puede ver afectado por la inestabilidad financiera. En contraste, la estabilidad financiera puede tener una contribución positiva al crecimiento económico de estos países (Neaime y Gaysset, 2018). Ya que para evitar la ocurrencia de estas crisis financieras es necesario un sistema financiero estable e incluyente, el cual sea beneficioso para la sociedad. En general, la estabilidad financiera puede afectar al crecimiento de manera positiva a través de tres canales: menor incertidumbre, menor volatilidad y menores costos de financiamiento (Carbó y Pedagua, 2013). Para medir los efectos de la estabilidad sobre el crecimiento económico se consideran tres variables las cuales se enfocan en la medición 
de la incertidumbre estas son: la razón préstamos en mora a prestamos brutos en el sector bancario, la proporción del crédito bancario a depósitos bancarios y por último la proporción de activos líquidos a depósitos y fondeo a corto plazo en el sector bancario.

Por otro lado, en la literatura de la medición de la inclusión financiera se han considerado tres diferentes dimensiones para evaluar la inclusión financiera, estas son: alcance del crédito, alcance de los depósitos y alcance de las sucursales. Estos indicadores se emplean tanto en estudios de corte transversal para varios países como a nivel individual (Sethi y Acharya, 2018). Algunos ejemplos de estos trabajos son los de: Sarma (2008), Arora (2010), Sarma y Pais (2010), Chattopadhyay (2011), Ghosh (2011), Sarma (2012), Sethy (2015), Sharma (2016), Sethi y Sethy (2019), Sethi y Acharya (2018) y Kim, Yu y Hassan (2018)

En conjunto inclusión y estabilidad financiera, son dimensiones del sistema financiero de suma importancia para su apropiado funcionamiento. Por lo anterior, estudiar la relación entre inclusión financiera y crecimiento económico al igual que el nexo estabilidad financiera-crecimiento económico es de vital importancia. El presente estudio analiza estas relaciones bajo dos hipótesis: la primera es que la inclusión financiera tiene un efecto positivo sobre el crecimiento económico y la segunda hipótesis es que la estabilidad financiera tiene un efecto positivo sobre el crecimiento económico. Para probar estas conjeturas se emplea una muestra de 71 países para el período 2006-2017. Se utilizan dos métodos para realizar la estimación: el primero es el de Mínimos Cuadrados Ordinarios (MCO) con efectos fijos de dos vías y el segundo es el Método Generalizado de Momentos (GMM) con efectos fijos de dos vías. Se elige el método de Mínimos Cuadrados Ordinarios con efectos fijos de dos vías para considerar los efectos no observables específicos de cada país en la muestra, al igual que los efectos no observables en el tiempo. A diferencia de otros trabajos como los de Sethi y Sethy (2019), Sethi y Acharya (2018) y Kim, Yu y Hassan (2018) que emplean como medida del desempeño económico el Producto Interno Bruto per cápita, en la presente investigación se utiliza la tasa de crecimiento anual en porcentaje del Producto Interno Bruto.

El resto del estudio se organiza de la siguiente forma: la sección dos contiene la revisión de la literatura y el marco teórico, la sección tres incluye el análisis descriptivo de los datos utilizados, las secciones cuatro y cinco explican la metodología y los resultados. Por último, en la sección seis se discuten las conclusiones y las posibles futuras investigaciones.

\section{Revisión de la literatura}

La evidencia empírica reciente del nexo entre desarrollo financiero y crecimiento apoya la hipótesis de McKinnon (1973), esta sugiere que un flujo de fondos canalizado a través de un sistema financiero eficiente ayuda a acelerar el crecimiento económico. En este sentido, 
los bancos son las instituciones que están en una mejor posición para ayudar a fortalecer el sistema financiero de una economía. Sin embargo, cuando una parte importante de la población no tiene acceso al sistema financiero, el escenario cambia. Ya que esta parte de la población (que no tiene acceso al sistema financiero formal e informal), no puede acceder a servicios: como depósitos y créditos (Sharma, 2016). La inclusión financiera puede contribuir al crecimiento económico en dos formas. La primera forma es a través de proveer acceso asequible a los servicios financieros para los sectores más desfavorecidos de la sociedad lo cual reduce la vulnerabilidad de los mismos y además mejora sus estándares de vida (Rajan, 2009). Esto se logra con créditos de bajo costo a personas de bajos ingresos y grupos vulnerables esto promueve el inicio de las actividades organizadas, lo cual aumenta la producción. Esta adición en valor promueve el crecimiento económico a nivel estatal y nacional (Sethi y Acharya, 2018). Lo cual significa mejores niveles de vida para estos grupos vulnerables al aumentar los niveles de ingreso de estas personas. De igual manera, esta situación disminuye la pobreza en las áreas rurales mientras promueven el crecimiento económico. Segundo el acceso universal a productos financieros como depósitos y seguros para las personas excluidas presenta los siguientes beneficios: aumentan los fondos en los mercados financieros, permite a las personas ahorrar en las instituciones del sistema financiero formal y a su vez estos mercados financieros aseguran la colocación eficiente de estos fondos en proyectos de inversión de largo plazo, ayudan a los individuos a cubrirse contra el riesgo de liquidez, el cual es provocado por la escasez de fondos en el mercado. Cuando los individuos pueden cubrirse contra el riesgo de liquidez, da como resultado mayor producción y más empleos lo que conlleva a una mejora en la distribución del ingreso y a un mayor ingreso en el sector de la población con menos recursos (Claessens y Perotti, 2007).

Por otro lado, se ha notado que hay tres diferentes canales a través de los cuales la estabilidad financiera puede afectar al crecimiento económico. El primer canal sugiere que la incertidumbre disminuye cuando existe estabilidad acerca del valor fundamental de los activos, esto hace que los inversionistas exhiban un comportamiento más proclive a mayores niveles de inversión cuando existe estabilidad financiera. El segundo canal en que la estabilidad financiera puede apoyar al crecimiento es que cuando existe estabilidad, la volatilidad de los precios de los activos es baja, lo que provoca que las empresas inviertan más en estos períodos. Un tercer canal se da cuando existe estabilidad financiera, esto hace que las condiciones de préstamo mejoren, esto permite que las personas pueden acceder más fácilmente al crédito, lo que tiene un efecto positivo sobre el crecimiento económico. Así mismo cuando existe estabilidad financiera el costo de financiamiento disminuye lo que significa costos más bajos de sus gastos financieros para hogares y empresas, esta disminución en los costos financieros casusa que los hogares y empresas gasten más, esto trae como resultado mayor crecimiento económico (Carbó y Pedauga, 2013). 
En la literatura el término inclusión financiera ha recibido mayor atención desde finales de la década de los noventa y la investigación en este período se enfoca sobre todo en el tipo de personas que están excluidas del sistema financiero. A inicios del año 2000 los estudios abordaban principalmente la definición de inclusión financiera y las características de las personas excluidas financieramente. A partir de 2005 la investigación se centra en la medición de la inclusión financiera y su relación con el desarrollo económico (Kim, Yu y Hassan, 2018). Entre los trabajos más recientes que tratan sobre la medición de la inclusión financiera destacan los trabajos de: Sarma (2008), Arora (2010), Demirguc-kunt y Klapper (2012), Yorulmaz (2013), Park y Mercado (2018) y Sethi y Sethy (2019).

En el trabajo de Sarma (2008) se propone un índice multidimensional para analizar la inclusión financiera de una manera integral. Siguiendo la metodología de Sarma (2008) para medir el acceso a los servicios financieros; en el trabajo de Arora (2010) se calcula el índice de inclusión financiera para países desarrollados y en desarrollo. Mientras que en el trabajo de Demirguc-kunt y Klapper (2012) se hace el primer análisis que emplea la base de datos Global de Inclusión Financiera, esta base mide como las personas solicitan créditos, ahorran y administran el riesgo. Ellos analizan el comportamiento del uso de los servicios financieros en 148 países.

Yorulmaz (2013) desarrolla un índice de inclusión financiera el cual hace uso de tres dimensiones para medir la cobertura de los servicios financieros para el caso de Turquía. En este estudio se usa el método de Sarma (2008) para crear índices de inclusión financiera y se encuentra que las regiones de alto ingreso tienden a mostrar mayores niveles de inclusión financiera y viceversa. En este sentido Park y Mercado (2018) presentan un nuevo índice de inclusión financiera, ellos utilizan el método de componentes principales para calcular los pesos de los nueve indicadores que componen su índice. Estos se clasifican en indicadores de acceso, disponibilidad y uso. Además, analizan el impacto de la inclusión financiera sobre: la pobreza, desigualdad del ingreso, emprendimiento y el empoderamiento de la mujer. Y encuentran evidencia robusta de que los países con altos niveles de inclusión financiera tienen menores tasas de pobreza, se realiza más emprendimiento y se registra mayor empoderamiento de la mujer. Por último, en el trabajo de Sethi y Sethy (2019) también se hace uso de la metodología de Sarma (2008) para crear un índice de inclusión financiera. Lo anterior con el fin de realizar un análisis de cointegración lineal y no lineal entre inclusión financiera y crecimiento económico para el caso de la India, ellos utilizan el enfoque de rezago distribuido auto regresivo (ARDL), además, ellos realizan la prueba de causalidad de Granger utilizando el enfoque de Toda-Yamamoto. La prueba de cointegración lineal confirma una relación de largo plazo entre inclusión financiera y crecimiento económico. Sin embargo, no se encuentra evidencia de una cointegración no lineal. Así mismo, los resultados de la prueba de causalidad muestran que la inclusión financiera causa en el sentido de Granger al crecimiento económico. 
Por otro lado, para la relación entre inclusión financiera y crecimiento económico se presentan algunos trabajos que aportan evidencia empírica a favor de un nexo positivo entre inclusión financiera y crecimiento económico estos son: Acharya, Amanulla y Joy (2009), Prasad (2010), Ellis, Lemma y Rudd (2010), Ghosh (2011), Diniz, Birochi y Pozzebon (2012), Dupas y Robinson (2013), Sharma (2016), Pradhan, Arvin, Hall y Nair (2016), Kim, Yu y Hassan (2018) y Sethi y Acharya (2018). En el trabajo de Acharya, Amanulla y Joy (2009) se confirma una relación de cointegración de largo plazo entre crecimiento del crédito y crecimiento del producto en los estados de la India; el estudio analiza estados considerados avanzados y atrasados económicamente de la India para el período 1981-2002. De igual manera Prasad (2010) apunta a que la falta de acceso al sistema financiero formal tiende a disminuir el crecimiento económico y el bienestar de las personas. De hecho, la falta de acceso adecuado al crédito por parte de empresas y emprendedores en el sector de servicios tiene un efecto adverso sobre el nivel de empleo, ya que las empresas en este sector tienden a hacer un uso intensivo de mano de obra en sus operaciones. En este mismo sentido, Ellis, Lemma y Rudd (2010) encuentran que el acceso al sistema financiero fomenta la inversión en los hogares, lo cual a su vez provoca mayor crecimiento económico.

En un análisis a nivel estatal, Ghosh (2011) observa que mayor inclusión financiera se traduce en un mayor Producto Interno Bruto per cápita en la India. Así mismo, Diniz, Birochi y Pozzebon (2012) observan que proporcionar servicios bancarios por correo ha traído un cambio social y económico a nivel local en la región del Amazonas. En el trabajo de Dupas y Robinson (2013), se demuestra que proveer a los individuos con instrumentos de ahorro, incrementa la inversión productiva en la economía. En un estudio más reciente, Sharma (2016) encuentra que indicadores como: el número de cuentas para préstamo y depósito, así como el alcance demográfico de los cajeros automáticos tienen una causalidad unidireccional con el crecimiento económico. En el trabajo de Pradhan, Arvin, Hall y Nair (2016) se estudia la relación entre la penetración del mercado de seguros y la inclusión financiera. Además, estudian la interacción de la causalidad entre la penetración del mercado de seguros, dinero, capitalización del mercado de acciones y crecimiento económico, se enfocan en la asociación de naciones del sudeste asiático. Los resultados, revelan que las variables están cointegradas y se causan mutuamente. Concluyen que existe una causalidad bidireccional de corto plazo entre el mercado de seguros y el crecimiento económico. Usando una muestra de 55 países que pertenecen a la Organización de Cooperación Islámica, Kim, Yu y Hassan (2018) examinan la relación entre inclusión financiera y crecimiento. Utilizan dos métodos de estimación para panel de datos, los cuales son: GMM y el método de Vectores Autorregresivos (VAR). Y sus resultados muestran que la inclusión financiera tiene un efecto significativo sobre el crecimiento económico, además de que el análisis de causalidad de Granger arroja una causalidad mutua entre inclusión financiera y crecimiento económico. Para analizar el impacto 
dinámico de la inclusión financiera sobre el crecimiento económico Sethi y Acharya (2018), utilizan diferentes técnicas econométricas de panel de datos como: modelos de efectos fijos, cointegración de panel, modelos de efectos aleatorios y pruebas de causalidad. La muestra está compuesta por 31 países. Ellos encuentran que existe una relación positiva de largo plazo entre inclusión financiera y crecimiento económico, además de que la prueba de causalidad muestra una relación bilateral entre inclusión financiera y crecimiento económico.

Por el contrario, de acuerdo al trabajo de Mehrotra y Yetman (2015) podría no existir el nexo positivo entre la inclusión financiera y el crecimiento económico, ya que ellos argumentan que cuando se alcanza la inclusión financiera a través de un aumento rápido del crecimiento del crédito o a través de intermediarios financieros no regulados, esto puede afectar la estabilidad financiera y como consecuencia puede resultar en un nexo negativo entre inclusión financiera y crecimiento económico.

La estabilidad financiera del sistema financiero es crucial, porque permite el flujo de dinero entre individuos, esta situación permite a los individuos consumir e invertir, lo que a su vez promueve mayor crecimiento económico (Alsamara, Mrabet, Jarallah y Barkat, 2018). Por tanto, la estabilidad financiera se define como la característica del sistema financiero, que asegura de manera permanente y sin alteraciones una eficiente colocación de los recursos financieros de acuerdo con Mishkin (1992).

Batuo, Mlambo y Asongu (2018) estudian la relación entre inestabilidad financiera, liberalización financiera, desarrollo financiero y crecimiento económico para el caso de 41 países de África. Sus resultados sugieren que el desarrollo del sistema financiero y la liberalización financiera tienen un efecto positivo sobre la inestabilidad. Así mismo encuentran que el crecimiento económico reduce la inestabilidad financiera. Aboura y van Roye (2017) desarrollan un índice de estrés financiero para medir en tiempo real el estado de la estabilidad financiera. Usan el método de Auto Regresiones Vectoriales Bayesianas de Conmutación de Markov (MS-BVAR) para mostrar que cuando se presentan elevados niveles de estrés financiero, estos se asocian con menor actividad económica. En este mismo sentido Duprey, Klaus y Peltonen (2017) también desarrollan un índice de estrés financiero para 27 países de la Unión Europea. Sus resultados muestran que episodios de estrés financiero se asocian a un substancial impacto negativo en la economía. Para analizar el nexo entre estabilidad financiera y desempeño económico en la Unión Europea Creel, Hubert y Labondance (2015), utilizan el método GMM considerando el período 1988-2011. Sus resultados sugieren que la inestabilidad financiera afecta negativamente al crecimiento económico.

Estudiando la interacción entre competencia en el sector bancario, estabilidad financiera y crecimiento económico, el trabajo de Jayakumar, Pradhan, Dash, Maradana y Gaurav (2018) se enfocan en la dirección de la causalidad de Granger. El método econométrico empleado es el Modelo Vector Error-Corrección (VECM). Los resultados empíricos muestran que tanto 
la competencia bancaria como la estabilidad financiera son indicadores significativos que impulsan el crecimiento económico en los países miembros de la Unión Europea. Para el caso de Qatar en el período 1980-2013 Alsamara, Mrabet, Jarallah y Barkat (2018) investigan la relación entre estabilidad financiera y crecimiento económico. Ellos estiman el impacto de corto y largo plazo del crecimiento económico sobre la provisión de préstamos utilizando el Modelo Vector Error-Corrección con cambios estructurales. Ellos encuentran que el crecimiento económico tiene una relación de largo plazo negativa sobre la provisión de préstamos y una relación positiva de corto plazo sobre el suministro de préstamos.

\section{Datos}

Este estudio explora la relación entre inclusión financiera y crecimiento económico para una muestra de 71 países en el período 2007-2016. Los datos para las variables que miden la inclusión y estabilidad financiera son tomados de la base de datos Global de Desarrollo Financiero elaborada por el Banco Mundial, Mientras que las variables de control son tomadas de la base de datos Indicadores de Desarrollo Mundial realizada por el Banco Mundial.

Para medir el grado de inclusión financiera se utilizan dos variables: la primera es el número de cajeros automáticos por cada 100,000 habitantes y la segunda medida es el número de sucursales bancarias por cada 100,000 adultos. El número de cajeros automáticos por cada 100,000 habitantes mide la propiedad de las cuentas, se asume que la propiedad de las cuentas indica cuantas personas o empresas tienen cuentas en las instituciones financieras formales. Aunque la mejor forma de medir este factor clave es contar el número de personas o firmas que tienen cuentas, los datos no están disponibles. Por tanto, se usa el número de cajeros automáticos como aproximación de la propiedad de cuentas porque las instituciones financieras generalmente emiten una tarjeta de débito cuando se abre una cuenta, entonces la tasa de penetración de cajeros automáticos representará indirectamente la tasa de penetración de cuentas bancarias. La segunda medida es el número de sucursales bancarias por cada 100,000 adultos como una aproximación de la tasa de penetración de las instituciones financieras. Se elige esta variable porque es posible inferir el grado de predominio de las instituciones financieras a través del número de sucursales en un país (Kim, Yu y Hassan 2018). La variable utilizada para medir el desempeño económico es el incremento anual porcentual del Producto Interno Bruto.

Por otro lado, para analizar la relación entre crecimiento económico y estabilidad financiera, se utiliza la razón préstamos en mora a prestamos brutos en el sector bancario. Está variable mide la proporción de los créditos sobre los cuales no se ha realizado el pago programado en un tiempo específico respecto al total de créditos otorgados. Un aumento de 
esta variable significa un aumento en la probabilidad de bancarrota, por tanto, es un indicador de inestabilidad financiera. De igual manera se emplea la proporción del crédito bancario a depósitos bancarios esta variable evalúa la liquidez del sector bancario. Y consiste en la relación de la cantidad de los depósitos que se ha movilizado a préstamos. Si esta proporción es muy alta, significa que en el sector bancario puede no tener suficiente liquidez para cubrir cualquier requerimiento de fondos no previsto. Por otra parte, si la proporción es muy baja entonces los bancos no están obteniendo tantas ganancias como deberían. Niveles altos para las variables y significan mayor incertidumbre mientras que niveles más bajos de estas variables significan menor incertidumbre.

Por último, la proporción de activos líquidos a depósitos y fondeo a corto plazo indica hasta qué grado la liquidez disponible de los bancos está soportada por fondos propios y préstamos a corto plazo. Mide que tan vulnerable es un banco si estas fuentes de financiamiento disminuyen. Un porcentaje alto de esta variable sugiere un bajo nivel de vulnerabilidad al riesgo de liquidez, es decir existe una menor probabilidad de bancarrota. Por el contrario, un porcentaje bajo de esta variable sugiere un alto nivel de riesgo de liquidez, lo que implica mayor probabilidad de bancarrota y mayor incertidumbre

De igual manera, se consideran dos tipos de variables de control: macroeconómicas y de ambiente regulatorio. Las variables de control macroeconómicas son las siguientes: mide el comercio internacional se espera que su signo sea positivo, es el índice de precios al consumidor se espera que su signo sea negativo, es la formación bruta de capital se espera que el signo sea positivo. Las variables de control del ambiente regulatorio son: es la calidad regulatoria se espera que el signo sea positivo y es el estado de derecho se espera que el signo sea positivo. Tanto como tienen un rango de valores -2.5 a 2.5 , los valores más altos implican mejor desempeño. 
Tabla 1

Resumen de variables y fuentes

\begin{tabular}{|c|c|c|}
\hline Variable & Definición & Fuente \\
\hline growth & $\begin{array}{l}\text { Tasa de incremento anual en porcentaje } \\
\text { del Producto Interno Bruto }\end{array}$ & Global Financial Development Database \\
\hline atm & $\begin{array}{l}\text { Numero de cajeros automáticos por cada } \\
100,000 \text { habitantes }\end{array}$ & Global Financial Development Database \\
\hline branches & $\begin{array}{l}\text { Número de sucursales bancarias por cada } \\
100,000 \text { adultos }\end{array}$ & Global Financial Development Database \\
\hline non performing & $\begin{array}{l}\text { Razón préstamos en mora a prestamos } \\
\text { brutos }\end{array}$ & Global Financial Development Database \\
\hline credit deposits & $\begin{array}{l}\text { Proporción del crédito bancario a depósi- } \\
\text { tos bancarios }\end{array}$ & Global Financial Development Database \\
\hline liquid assets & $\begin{array}{l}\text { Proporción de activos líquidos a depó- } \\
\text { sitos y fondeo a corto plazo en el sector } \\
\text { bancario }\end{array}$ & Global Financial Development Database \\
\hline trade & $\begin{array}{l}\text { Es la suma de las exportaciones e impor- } \\
\text { taciones, medido como una proporción } \\
\text { del Producto Interno Bruto }\end{array}$ & World Develpment Indicators \\
\hline inflation & $\begin{array}{l}\text { Índice de precios al consumidor de } \\
\text { diciembre }\end{array}$ & Global Financial Development Database \\
\hline gross capital formation & $\begin{array}{l}\text { Consiste en desembolsos por adiciones a } \\
\text { los activos fijos de la economía más los } \\
\text { cambios netos en el nivel de inventarios }\end{array}$ & World Develpment \\
\hline regulatory quality & $\begin{array}{l}\text { Refleja las percepciones de la capacidad } \\
\text { del gobierno para formular e implementar } \\
\text { políticas y reglamentaciones sólidas que } \\
\text { permitan y promuevan el desarrollo del } \\
\text { sector privado }\end{array}$ & Worlwide Governance Indicators \\
\hline rule of law & $\begin{array}{l}\text { Refleja las percepciones de hasta qué pun- } \\
\text { to los agentes confían y respetan las reglas } \\
\text { de la sociedad }\end{array}$ & Worlwide Governance Indicators \\
\hline
\end{tabular}

Fuente: Elaboración propia. 
Tabla 2

Estadísticas descriptivas

\begin{tabular}{ccccccc}
\hline Variable & Obs. & $\begin{array}{c}\text { Unidad de } \\
\text { Medida }\end{array}$ & Media & $\begin{array}{c}\text { Desviación } \\
\text { estándar }\end{array}$ & Mínimo & Máximo \\
\hline growth & 710 & $\%$ & 5 & 12.48 & -33 & 48 \\
atm & 710 & Número & 63.65 & 46.16 & .29 & 299.77 \\
branches & 710 & Número & 24.04 & 24.22 & .48 & 257.7 \\
credit deposits & 710 & $\%$ & 122.03 & 85.80 & 18.34 & 879.66 \\
non performing & 710 & $\%$ & 5.76 & 6.23 & .06 & 48.68 \\
liquid assets & 710 & $\%$ & 29.92 & 14.15 & 5.27 & 127.97 \\
trade & 710 & $\%$ & 100.12 & 64.16 & 22.11 & 441.60 \\
inflation & 710 & $\%$ & 107.83 & 25.40 & 63.01 & 432.91 \\
gross capital formation & 710 & $\%$ & 23.92 & 6.15 & 9.82 & 55.36 \\
regulatory quality & 710 & Número & .5 & .79 & -1.43 & 2.26 \\
rule of law & 710 & Número & .32 & .94 & -1.37 & 2.10
\end{tabular}

Fuente: Elaboración propia.

\section{Metodología}

Para poder examinar la relación entre inclusión financiera y crecimiento económico, así como el posible nexo entre estabilidad financiera y crecimiento económico. Primero se establece el modelo de regresión para panel de datos, en este se emplea a la variable crecimiento económico como variable dependiente y a las variables inclusión y estabilidad financiera como variables independientes. Además, se incluyen las variables de control. Por tanto, el modelo se especifica de la siguiente forma:

$$
\begin{gathered}
\text { crecimiento }_{i, t}=c+\text { inclusion financiera }_{i, t}+\text { estabilidad financiera }_{i, t} \\
+ \text { variables de control } \\
i, t \\
+\epsilon_{i, t}
\end{gathered}
$$


Donde $i$ representa el país y $t$ representa el tiempo. La muestra que se utiliza considera 71 países en un período que abarca desde el año 2006 al año 2017. Para estos países existen características específicas que no podemos observar directamente. Así mismo existen ciertos factores que ocurren en el tiempo como huelgas, desastres naturales que tampoco se pueden observar directamente. Por estas razones se considera un modelo de efectos fijos de dos vías el cual considera la inclusión de dos variables dummies una para las características específicas de cada país y otra para los efectos del tiempo. El modelo se expresa de la siguiente forma:

$$
y_{i, t}=\theta_{i}+\tau_{t}+\beta_{1} x_{i, t}+\beta_{2} C_{i, t}+\varepsilon_{i t}
$$

Donde $\theta_{i}$ y $\tau_{\mathrm{t}}$ son los características específicas de cada país y los efectos temporales respectivamente, $x_{i, t}$ incluye las variables de inclusión y estabilidad financiera $C_{i, t}$ son las variables de control. Por tanto, el modelo a estimar es el siguiente:

$$
\begin{aligned}
& \text { growth }_{i, t}=\text { atm }_{i, t}+\text { branches }_{i, t}+\text { credit deposits }_{i, t}+\text { non performing } \text { fr,t }+
\end{aligned}
$$

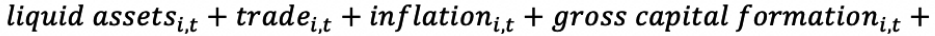

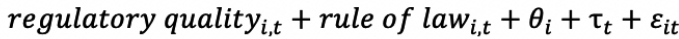

Donde $\epsilon_{i, t}$ es el término de error.

La prueba de especificación utilizada para determinar si se deben considerar efectos fijos u aleatorios, es la prueba de Hausman de Efectos Aleatorios Correlacionados. Además, como prueba de robustez se estima la ecuación (3) utilizando el Método Generalizado de Momentos (GMM) propuesto por Arellano y Bond (1991). El estimador GMM se describe en la siguiente ecuación:

$$
y_{i, t}=\beta y_{i, t-1}+\gamma x_{i, t}+\delta z_{i, t}+\vartheta w_{i, t}+\varepsilon_{i t}
$$

Donde $\mathrm{x}_{\mathrm{i}, \mathrm{t}}$ son las variables de control, $\mathrm{z}_{\mathrm{i}, \mathrm{t}}$ son las variables explicativas de estabilidad financiera $\mathrm{y}_{\mathrm{i}, \mathrm{t}}$ son las variables explicativas de inclusión financiera $\mathrm{y}_{\mathrm{i}, \mathrm{t}}$ es la variable de desempeño económico y $\beta y_{i, t-1}$ representa su valor con rezago. Arellano y Bond (1991) recomiendan el uso de variables de las variables explicativas con rezago como variables instrumentales. La consistencia de este estimador depende de la validez de los instrumentos. Para abordar esta situación se utiliza la prueba de especificación Hansen Sargan de sobre identificación de restricciones, la cual examina la validez general de los instrumentos al analizar la muestra analógica de las condiciones de momento utilizadas en el proceso de estimación. Se utiliza 
este estimador con una modificación la cual consiste en no considerar el término $\beta y_{i, t-1}$ haciendo el modelo no dinámico.

De igual manera para poder examinar la dirección de la causalidad entre las variables de la ecuación, se estima la prueba de causalidad de Granger. La prueba de Granger de causalidad para panel de datos puede ser estimada de dos formas. El primer método es tratar el panel como un conjunto de datos apilados, en el que se supone que todos los coeficientes son comunes en todas las secciones transversales. Este método asume que todos los coeficientes son iguales sin considerar las diferencias en el corte transversal y el panel de datos se toma como una serie de tiempo ordinaria. El segundo método consiste en estimar la prueba de Granger bajo el supuesto de que lo coeficientes no son iguales en todas las secciones transversales, este método fue propuesto por Dumitresu y Hurlin (2012). Dumitrescu y Hurlin (2012) desarrollan una prueba extendida la cual está diseñada para detectar causalidad en panel de datos. Este método calcula la prueba de causalidad estándar de Granger para cada panel y también calcula un estadístico zbar del promedio de las pruebas estadísticas. Se realiza la prueba de causalidad de Granger desarrollada por Dumitrescu y Hurlin (2012) con este método debido a que, no existen suficientes datos en la muestra para realizar la prueba con el primer método. La regresión subyacente de esta prueba se expresa de la siguiente forma:

$$
y_{i, t}=\alpha_{i}+\sum_{k=1}^{K} \beta_{i k} y_{i, t-k}+\sum_{k=1}^{K} \gamma_{i k} x_{i, t-k}+\varepsilon_{i, t}
$$

Donde $y_{i, t}$ y $x_{i, t}$ son las observaciones de dos variables estacionarias para el individuo en el período .A los coeficientes se les permite variar entre individuos, pero se asume que no varían en el tiempo. El orden de rezago $K$ se asume idéntico para todos los individuos, además el panel debe estar balanceado. Como en Granger (1969) el procedimiento para determinar la existencia de causalidad es probar por efectos significativos en los valores pasados de sobre los valores presentes de $y$.

\section{Resultados}

Los resultados de la estimación empleando el método de Mínimos Cuadrados Ordinarios con efectos fijos de dos vías se pueden observar en la tabla 3. Para las variables que miden inclusión financiera se obtuvieron los siguientes resultados: el signo del coeficiente de la variable es negativo y es estadísticamente significativa al 5\%, esto significa que un aumento del $1 \%$ 
del número de cajeros automáticos por cada 100,000 adultos se traduce en una disminución del crecimiento del $.06 \%$. La variable branches tiene signo negativo y es estadísticamente significativa al 1\%, un aumento en esta variable significa una disminución del crecimiento de $.07 \%$. Por otro lado, los resultados para las variables que evalúan la estabilidad financiera muestran que la variable credit deposits exhibe signo negativo y es estadísticamente significativa al $5 \%$, un aumento de $1 \%$ de esta variable significa una disminución del crecimiento de $.03 \%$ del crecimiento económico. Mientras que las variables non performing y liquid assets no son estadísticamente significativas. En cuanto a las variables de control, estas exhiben los siguientes resultados: inflation tiene el signo esperado y la variable es estadísticamente significativa al $1 \%$. Por otro lado, la variable gross capital formation presenta signo positivo el cual es el esperado y es estadísticamente significativo al 1\%. Mientras que las variables trade, regulatory quality, y rule of law no son estadísticamente significativas. Los resultados para la prueba de Hausman de Efectos Aleatorios rechazan la hipótesis nula de la no existencia de una especificación errónea, por tanto, se utilizan efectos fijos.

Los resultados de la estimación empleando el Método de Momentos Generalizados (GMM) con efectos fijos de dos vías muestran que la variable no es estadísticamente significativa, el coeficiente de la variable exhibe signo negativo y es estadísticamente significativo al $10 \%$. En cuanto a las variables que analizan la estabilidad financiera los resultados son los siguientes: el coeficiente de la variable tiene signo negativo esto significa que un aumento de $1 \%$ en la variable se refleja en una disminución del $.07 \%$ en el crecimiento económico, la variable tiene signo positivo y es estadísticamente significativa al 5\%. En cuanto a la variable no es estadísticamente significativa. Las variables de control y no son estadísticamente significativas. En cuanto a las variables e , las dos variables cuentan con el signo esperado; positivo para, y signo negativo para. Además ambas son estadísticamente significativas es estadísticamente significativa al 1\%, mientras que es estadísticamente significativa al $5 \%$. Los resultados de la prueba Hansen Sargan validan las variables instrumentales utilizadas.

Los resultados de ambas estimaciones apuntan a que existe evidencia de una relación negativa entre inclusión financiera y crecimiento económico. Así mismo, se encuentra evidencia de una relación negativa entre estabilidad financiera y crecimiento económico cuando se considera la variable. Mientras que la variable exhibe evidencia de una relación positiva con la variable crecimiento económico para el modelo estimado con el método GMM. 
Tabla 3

Resultados de la estimación con el método de efectos fijos de dos vías y el Método de Momentos Generalizados con efectos fijos de dos vías

\begin{tabular}{|c|c|c|}
\hline Coeficientes & $\begin{array}{c}\text { MCO con efectos fijos de dos } \\
\text { vías (1) }\end{array}$ & $\begin{array}{c}\text { GMM con efectos fijos de dos } \\
\text { vías. (2) }\end{array}$ \\
\hline \multicolumn{3}{|l|}{ Variable dependiente } \\
\hline \multicolumn{3}{|l|}{ growth } \\
\hline \multicolumn{3}{|l|}{ Variable independiente } \\
\hline$c$ & $0.171593 * * *$ & -0.052585 \\
\hline atm & $-0.000680 * *$ & -0.000466 \\
\hline branches & $-0.000753 * * *$ & $-0.000570 *$ \\
\hline credit deposits & $-0.000334^{* *}$ & $-0.000711^{* *}$ \\
\hline non performing & -0.001503 & $0.003270^{* *}$ \\
\hline liquid assets & .0000643 & 0.000144 \\
\hline \multicolumn{3}{|l|}{ Variable de control } \\
\hline trade & -0.000146 & $0.002262 * * *$ \\
\hline inflation & $-0.000673 * * *$ & $-0.000527 * *$ \\
\hline gross capital formation & $0.003910 * * *$ & -0.000251 \\
\hline regulatory quality & -0.021904 & -0.003296 \\
\hline rule of law & -0.014704 & 0.063938 \\
\hline \multicolumn{3}{|l|}{ Estadísticas } \\
\hline Observaciones & 710 & \\
\hline$R$-cuadrado & 0.656326 & 568 \\
\hline Durbin- Watson & 2.043500 & 0.560150 \\
\hline Estadístico J & ----------- & 2.034556 \\
\hline Probabilidad Estadistico $J$ & ----------- & 0.300819 \\
\hline Probabilidad Efectos & & 0.583369 \\
\hline Aleatorios Correlacionados & .0000 & \\
\hline
\end{tabular}

Fuente: Elaboración propia. 
Tabla 4

Resultados de la prueba apilada de causalidad de Granger

Prueba de causalidad de Granger por pares

Retrasos: 2

Observaciones: 588

Hipótesis Nula

Estadístico Probabilidad Observaciones

\begin{tabular}{|c|c|c|c|}
\hline no Causa Granger & 13.5533 & 2.E- $06 * * *$ & Unidireccional \\
\hline no Causa Granger & 3.18474 & 0.0421 & \\
\hline no Causa Granger & 11.0636 & 2.E- $05 * * *$ & Unidireccional \\
\hline no Causa Granger & 1.38772 & 0.2505 & \\
\hline no Causa Granger & 1.84047 & 0.1597 & Unidireccional \\
\hline no Causa Granger & 0.1597 & $0.0122 * *$ & \\
\hline no Causa Granger & 1.61864 & 0.1991 & Unidireccional \\
\hline no Causa Granger & 8.43163 & $0.0002 * * *$ & \\
\hline no Causa Granger & 0.17643 & 0.8383 & No hay \\
\hline no Causa Granger & 0.73135 & 0.4817 & Relación causal \\
\hline
\end{tabular}

Fuente: Elaboración propia.

Así mismo, se realizó la prueba de causalidad de Granger con el método de datos apilados con dos retrasos desarrollado por Dumitrescu y Hurlin (2012). Para las variables que miden la inclusión financiera se observa que la causalidad se extiende desde el número de cajeros automaticos por cada 100,000 adultos a la tasa de crecimiento del PIB de modo unidireccional, en cuanto a la relación causal para el número de sucursales bancarias por cada 
100,000 adultos esta se extiende del número de sucursales bancarias por cada 100,000 adultos hacia la tasa de crecimiento del PIB de manera unidireccional. En cuanto a las variables de estabilidad financiera, para la variable credit deposits la dirección de la relación causal se extiende desde growth a credit deposits de manera unidireccional, así mismo los resultados de la prueba apilada de causalidad Granger para la variable non performing muestra que la relación causal es unidireccional y se extiende desde growth a non performing. Por último las variables growth y liquid assets no tienen relación causal en el sentido de Granger de acuerdo a los resultados obtenidos en la prueba.

\section{Conclusiones}

Se realizaron dos estimaciones en la primera se empleó el método de efectos fijos de dos vías, mientras que en la segunda estimación se empleó el método GMM con efectos fijos de dos vías. La muestra utilizada consiste en 71 países en un período que abarca del año 2007 al año 2016. Los resultados muestran que las variables atm y branches exhiben signo negativo en ambas estimaciones y son estadísticamente significativas.

Estos resultados indican la existencia de evidencia a favor de una relación negativa entre acceso al sistema financiero y el crecimiento económico. Lo cual contradice la hipótesis inicial de este estudio de la existencia de una relación positiva entre inclusión financiera y crecimiento económico.

Los resultados obtenidos en la presente investigación no coinciden con los obtenidos en los trabajos de Sethi y Acharya (2018), Kim, Yu y Hassan (2018), Sharma (2016) y Sethi y Sethy (2019). A diferencia de estos trabajos los cuales utilizan como variable dependiente el PIB per cápita; en el presente análisis se emplea la tasa de crecimiento anual en porcentaje del Producto Interno Bruto de cada país. Por otro lado, los resultados de la prueba de causalidad de Granger muestran que la causalidad se extiende desde las variables y hacia . Y estos resultados sí son consistentes con los trabajos de Sharma (2016), Sethi y Acharya (2018), Kim, Yu y Hassan (2018) y Sethi y Sethy (2019).

Por otro lado, para analizar la relación entre estabilidad en el sector financiero y crecimiento se emplean la razón préstamos en mora a prestamos brutos y la proporción del crédito bancario a depósitos bancarios como medidas de inestabilidad. Además, se utiliza la proporción de activos líquidos a depósitos y fondeo a corto plazo en el sector bancario como medida de estabilidad.

Los resultados muestran que la variable credit deposits es estadísticamente significativa en ambos modelos y muestra signo negativo. Este resultado incida que mayor incertidumbre en 
el sistema financiero está relacionada con menor crecimiento económico. Por tanto, coincide con la hipótesis inicial de que mayor estabilidad en el sistema financiero estaría relacionada con mayor crecimiento económico.

En cuanto a la variable liquid assets no es estadísticamente significativa para ninguno de los modelos.

Mientras que la variable non performing es estadísticamente significativa en el modelo 2, y muestra un signo positivo. Esto significa que mayor incertidumbre en el sistema financiero está relacionada con mayor crecimiento económico. Este resultado aporta evidencia en contra de la hipótesis de la existencia de una relación positiva entre estabilidad financiera y crecimiento económico.

Los resultados de los trabajos de Aboura y van Roye (2017) Duprey, Klaus y Peltonen (2017) y Creel, Hubert y Labondance (2015) concuerdan con los resultados obtenidos en esta investigación.

La existencia de una relación positiva entre el aumento de la variable non performing y el aumento del crecimiento se puede originar en el momento en que los individuos dejan de pagar sus préstamos, y utilizan el dinero destinado a esos pagos al consumo lo cual estimula el crecimiento económico. Este resultado podría sugerir la creación de una política de suspensión de pagos temporal por parte del gobierno. Esta política consistiría en que los individuos con préstamos en mora durante una recesión económica podrían suspender de manera temporal el pago de los préstamos recibidos siendo el gobierno el encargado de pagar los intereses durante el tiempo que dure la recesión económica con el fin de estimular el crecimiento. O se podría incentivar la creación de un seguro en caso de recesión por parte del sistema financiero el cual cubra el pago de intereses de un préstamo durante una recesión para que los individuos aumenten su gasto en estos momentos. Esta política también se sustenta en base a los resultados de la prueba de causalidad de Granger dado que la relación causal va del crecimiento económico hacia la variable non performing.

Por otro lado, se sugiere la creación de un programa que condicione el acceso al sistema financiero a asistir y aprobar un curso de finanzas básicas, esto con dos objetivos el primero es condicionar el acceso al sistema financiero y el segundo es que los individuos que si tengan acceso al sistema puedan aprovechar sus ventajas.

Además, se proponen dos sugerencias para futuras investigaciones. La primera es analizar el impacto de la banca digital sobre la inclusión financiera. La segunda es investigar si la relación entre inclusión financiera es sensible al nivel de ingreso de los países. 


\section{Referencias}

Acharya, D. Amanulla, S. \& Joy, S. (2009), Financial development and economic growth in Indian states: an examination, International Research Journal of Finance and Economics, Vol. 24 (2), 117-130. (Disponible en: https://www.researchgate.net/publication/258117867_Financial_development_and_economie_growth_in_Indian_states_an_examination) (Consultado: 7/01/2019)

Aboura, S. \& van Roye, B. (2017). Financial stress and economic dynamics: The case of France. International Economics, 149, 57-73. http://dx.doi.org/10.1016/j.inteco.2016.11.001.

Alsamara, M., Mrabet, Z., Jarallah, S. \& Barkat, K. (2018). The switching impact of financial stability and economic growth in Qatar: Evidence from an oil-rich country. The Quarterly Review of Economics and Finance. http:// dx.doi.org/10.1016/j.qref.2018.05.008.

Angadi, V.B. (2003), Financial infrastructure and economic development: theory, evidence and experience. RBI Occasional Papers, 24 (1), pp. 191-223. (Disponible en: https://rbi.org.in/scripts/PublicationsView.aspx?Id=5908) (Consultado: 6/01/2019)

Arellano, M., \& Bond, S. (1991). Some Tests of Specification for Panel Data: Monte Carlo Evidence and an Application to Employment Equations. The Review of Economic Studies, 58(2), 277. http://dx.doi. org/10.2307/2297968

Arora, R. (2010), Measuring Financial Access, Discussion Papers in Economics. (Disponible en: https://www120. secure.griffith.edu.au/research/file/fd0408e2-75c5-c430-ea2d-2fe7274a467f/1/2010-07-measuring-financial-access.pdf) (Consultado: 24/01/2019)

Batuo, M., Mlambo, K., \& Asongu, S. (2018). Linkages between financial development, financial instability, financial liberalisation and economic growth in Africa. Research in International Business and Finance, 45, 168-179. http://dx.doi.org/10.1016/j.ribaf.2017.07.148.

Carbó, S. \& Pedagua, L. (2013). Financial Stability and Economic Growth. Crisis, Risk and Stability in Financial Markets, 8-23. http://dx.doi.org/10.1057/9781137001832_2.

Chattopadhyay, S. (2011) Financial Inclusion in India: A case-study of West Bengal. MPRA Paper 34269. (Disponible en: https://mpra.ub.unimuenchen.de/34269/1/MPRA_paper_34269.pdf) (Consultado: 23/01/2019)

Claessens, S. \& Perotti, E. (2007). Finance and inequality: Channels and evidence. Journal of Comparative Economics, 35(4), 748-773. http://dx.doi.org/10.1016/j.jce.2007.07.002.

Creel, J., Hubert, P. \& Labondance, F. (2015). Financial stability and economic performance. Economic Modelling, 48, 25-40. http://dx.doi.org/10.1016/j.econmod.2014.10.025.

Demirguc-Kunt, A. \& Klapper, L. (2012). Measuring Financial Inclusion: The Global Findex Database. Policy Research Working Papers. http://dx.doi.org/10.1596/1813-9450-6025.

Demirguc-Kunt, A., Klapper, L. \& Singer, D. (2017). Financial inclusion and inclusive growth : a review of recent empirical evidence. Policy Research working paper 8040. https://doi.org/10.1596/978-1-4648-1259-0

Demirguc-Kunt, A., Klapper, L. Singer D. \& Van Oudheusden. P. (2015). The Global Findex Database 2014: Measuring Financial Inclusion Around the World. World Bank Group. Policy Research Working Paper 7255. https:// doi.org/10.1596/1813-9450-7255

Diamond, D. \& Dybvig, P. (1983). Bank Runs, Deposit Insurance, and Liquidity. Journal of Political Economy, 91(3), 401-419. http://dx.doi.org/10.1086/261155.

Diniz, E., Birochi, R., \& Pozzebon, M. (2012). Triggers and barriers to financial inclusion: The use of ICT-based branchless banking in an Amazon county. Electronic Commerce Research and Applications, 11(5), 484-494. http://dx.doi.org/10.1016/j.elerap.2011.07.006.

Dumitrescu, E.-I., \& Hurlin, C. (2012). Testing for Granger non-causality in heterogeneous panels. Economic Modelling, 29(4), 1450-1460. http://dx.doi.org/10.1016/j.econmod.2012.02.014. 
Dupas, P. \& Robinson, J. (2013). Savings Constraints and Microenterprise Development: Evidence from a Field Experiment in Kenya. American Economic Journal: Applied Economics, 5(1), 163-192. http://dx.doi. org/10.1257/app.5.1.163.

Duprey, T., Klaus, B. \& Peltonen, T. (2017). Dating systemic financial stress episodes in the EU countries. Journal of Financial Stability, 32, 30-56. http://dx.doi.org/10.1016/j.jfs.2017.07.004.

Ellis, K., Lemma, A. \& Rudd, J. (2010). Financial inclusion, household investment and growth in Kenya and Tanzania. Project Briefing, 43, septiembre. (Disponible en: https:/www.odi.org/sites/odi.org.uk/files/odi-assets/ publications-opinion-files/5776.pdf) (Consultado: 20/01/2019)

Ghosh, S. (2011). Does financial outreach engender economic growth? Evidence from Indian states. Journal of Indian Business Research, 3(2), 74-99. http://dx.doi.org/10.1108/17554191111132206.

Goldsmith, R.W. (1969), Financial Structure and Development. (1 $\left.{ }^{\mathrm{a}} \mathrm{ed}\right)$. New Haven: Yale University Press.

Granger, C. W. J. (1969). Investigating Causal Relations by Econometric Models and Cross-spectral Methods. Econometrica, 37(3), 424. http://dx.doi.org/10.2307/1912791.

Greenwood, J. \& Jovanovic, B. (1990). Financial Development, Growth, and the Distribution of Income. Journal of Political Economy, 98(5). http://dx.doi.org/10.1086/261720.

Gurley, J. \& Shaw, E. (1955). Financial Aspects of Economic Development. The American Economic Review, 45(4), 515-538. (Disponible en: http://www.jstor.org/stable/1811632) (Consultado: 18/01/2019)

Jayakumar, M., Pradhan, R. P., Dash, S., Maradana, R. P. \& Gaurav, K. (2018). Banking competition, banking stability, and economic growth: Are feedback effects at work? Journal of Economics and Business, 96, 15-41. http://dx.doi.org/10.1016/j.jeconbus.2017.12.004.

Kim, D.-W., Yu, J.-S., \& Hassan, M. K. (2018). Financial inclusion and economic growth in OIC countries. Research in International Business and Finance, 43, 1-14. http://dx.doi.org/10.1016/j.ribaf.2017.07.178.

Mckinnon, R. I. (1973). Money and Capital in Economic Development. ( $\left.1^{\mathrm{a}} \mathrm{ed}\right)$. Washington,DC Brookings Institution Press.

Mehrotra, A. \& Yetman, J. (2015). Financial inclusion-issues for central Banks. BISQuarterly Review, marzo. (Disponible en: https://www.bis.org/publ/qtrpdf/r_qt1503h.htm) (Consultado: 11/12/2018)

Mishkin, F. S. (1992). Anatomy of a financial crisis. Journal of Evolutionary Economics, 2(2), 115-130. http:// dx.doi.org/10.1007/bf01193536.

Neaime, S. \& Gaysset, I. (2018). Financial inclusion and stability in MENA: Evidence from poverty and inequality. Finance Research Letters, 24, 230-237. http://dx.doi.org/10.1016/j.frl.2017.09.007.

Pradhan, R. P., Arvin, M. B., Hall, J. H. \& Nair, M. (2016). Innovation, financial development and economic growth in Eurozone countries. Applied Economics Letters, 23(16), 1141-1144. http://dx.doi.org/10.1080/13504851.2 016.1139668 .

Park, C.-Y. \& Mercado, R. (2018). Financial Inclusion: New Measurement and Cross-Country Impact Assessment. SSRN Electronic Journal. http://dx.doi.org/10.2139/ssrn.3199427.

Prasad, E. (2010). Financial Sector Regulation and Reforms in Emerging Markets: An Overview. http://dx.doi. org/10.3386/w16428.

Rajan, R.G. (2009). (1 ${ }^{\mathrm{a}}$ ed). A Hundred Small Steps - Report of the Committee on Financial Sector Reforms, New Delhi Planning Commission, Government of India, New Delhi. (Disponible en: http://planningcommission.nic. in/reports/genrep/rep_fr/cfsr_all.pdf) (Consultado: 12/01/2019)

Sarma, M. (2008). Index of Financial Inclusion, New Delhi Working Papers No. 215. (Disponible en: http://www. icrier.org/pdf/Working_Paper_215.pdf) (Consultado: 10/01/2019)

Sarma, M. (2012). Index of Financial Inclusion - a measure of financial sector inclusiveness. Competence Centre on Money, Trade, Finance and Development 1207. (Disponible en:https://finance-and-trade.htw berlin.de/fileadmin/HTW/Forschung/Money_Finance_Trade_Development/working_paper_series/wp_07_2012_Sarma_Index-of-Financial-Inclusion.pdf) (Consultado: 15/12/2018) 
Sarma, M. \& Pais, J. (2010). Financial Inclusion and Development. Journal of International Development, 23(5), 613-628. http://dx.doi.org/10.1002/jid.1698.

Sethi, D. \& Acharya, D. (2018). Financial inclusion and economic growth linkage: some cross country evidence. Journal of Financial Economic Policy, 10(3), 369-385. http://dx.doi.org/10.1108/jfep-11-2016-0073.

Sethy, S. K. (2015). Developing a Financial Inclusion Index and Inclusive Growth in India. The Indian Economic Journal, 63(2), 283-311. http://dx.doi.org/10.1177/0019466220150210.

Sethi, D. \& Sethy, S. K. (2019). Financial inclusion matters for economic growth in India. International Journal of Social Economics, 46(1), 132-151. http://dx.doi.org/10.1108/ijse-10-2017-0444.

Sharma, D. (2016). Nexus between financial inclusion and economic growth. Journal of Financial Economic Policy, 8(1), 13-36. http://dx.doi.org/10.1108/jfep-01-2015-0004.

Yorulmaz, R. (2013), "Construction of a regional financial inclusion index in Turkey”, Journal of BRSA Banking and Financial Markets, 7 (1). 79-101. (Disponible en: http://www.bddk.org.tr/ContentBddk/BddkDergi/dergi_0013_06.pdf) (Consultado: 15/01/2019)

\section{Anexo}

Tabla A5

\begin{tabular}{lll}
\hline Países & & \\
\hline Alemania & Francia & Namibia \\
Argentina & Federación Rusa & Noruega \\
Armenia & Georgia & Países Bajos \\
Australia & Grecia & Panamá \\
Austria & Guatemala & Paraguay \\
Bélgica & Honduras & Perú \\
Bielorrusia & Hungría & Polonia \\
Bolivia & Indonesia & Portugal \\
Bosnia y Herzegovina & Irlanda & República Checa \\
Brasil & Israel & República de Corea \\
Bulgaria & Italia & República Dominicana \\
Chile & Japón & República Kirguiza \\
Chipre & Jordán & República Eslovaca \\
Colombia & Kazajstán & Ruanda \\
Costa Rica & Letonia & Serbia \\
Croacia & Lituania & Singapur \\
Dinamarca & Luxemburgo & Sudáfrica \\
Ecuador & Macedonia & Suecia \\
Egipto & Malasia & Tanzania \\
El Salvador & Malta & Turquía \\
Eslovenia & Marruecos & Ucrania \\
España & México & Uruguay \\
Estonia & Moldavia & Vietnam \\
Filipinas & Mozambique & \\
\hline
\end{tabular}

Fuente: Elaboración propia. 